\title{
Developing a Gruyere-Like Trainer to Use in the Transition Between Basic and Neonatal Simulation-Based Minimally Invasive Surgery Education
}

\section{Maria M. Bailez, MD}

Division of Pediatric Surgery and Surgical Simulation Center, Garrahan Hospital, Buenos Aires, Argentina.

E-mail: mariamarcelabailez4@gmail.com

\section{Georgina Falcioni, MD}

Surgical Simulation Center, Garrahan Childrens Hospital, Buenos Aires, Argentina.

\section{Hsien Yang, MD}

Surgical Simulation Center, Garrahan Childrens Hospital, Buenos Aires, Argentina.

\section{Maximiliano Maricic, MD}

Surgical Simulation Center, Garrahan Childrens Hospital, Buenos Aires, Argentina.

Patricia Martinez

Surgical Simulation Center, Garrahan Childrens Hospital, Buenos Aires, Argentina.

Carolina Ruiz

Surgical Simulation Center, Garrahan Childrens Hospital, Buenos Aires, Argentina.

\section{Roxana Colombo}

Surgical Simulation Center, Garrahan Childrens Hospital, Buenos Aires, Argentina.

\section{Pablo Schiavo}

Telecommunication Office, Garrahan Childrens Hospital, Buenos Aires, Argentina.

(c) Mary Ann Liebert, Inc. DOI: 10.1089/vor.2019.0583
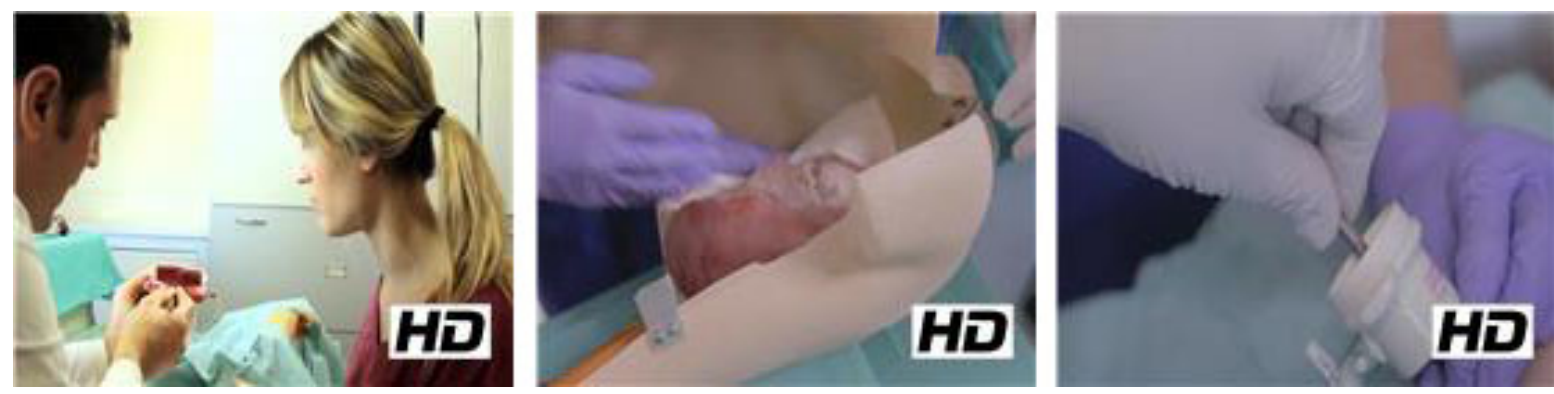

\section{Abstract}

Introduction: Minimal invasive training simulation laboratory has been developed to aid surgeons to overcome the challenging learning curve and included in our residents' curricula in 2012. Skills are trained in a programmed, progressive, and specific way, assessing performance. The first approach to endosurgery includes every aspect of minimally invasive surgery (MIS) such as knowledge of equipment and instruments, electrosurgery, ergonomy, and the art of MIS suturing. 
Aim: Present a versatile low-cost endotrainer for intermediate and advanced training in pediatric MIS before using the specific neonatal trainers.

Model: It consists in an adapted 10 year-old mannequin opened in half with the aid of hinges to maintain together. We call it "Gruyere" because of the multiple orifices strategically placed to perform abdominal pelvic procedures, esophageal and mediastinal procedures in the prone position and simulate left lobectomy in the lateral position. The retroperitoneal space was simulated and covered by an adhesive membrane. Commonly available components such as balloons, silicone, different type of fabrics, and siliconized vellon were used to simulate intra-abdominal pelvic organs, vessels, and nodes. Ex vivo porcine organs can also be used to resemble different procedures either in the abdomen or thorax. A simulated abdominal wall was created with synthetic materials such as sponge, cloth, nylon stockings, and silicone to resemble its components. This trainer allows the practice of trocar placement, lens management, and basic maneuvers as well as more complex procedures. Simulation of pelvic procedures such as ovarian cystectomy and lymphadenectomy can be achieved. Refined suturing practice can be done resembling an hepaticojejunostomy using porcine live tissue. We focus in training the whole team and the nurse role in handling the sutures to introduce them into the abdomen. We can see the practice of the shoeshine maneuver and suturing in a simulated Nissen. The prone position is meant to access the esophagus and mediastinum. Lymph nodes dissection is shown. A detailed tutorial of material, steps of each procedure and evaluation sheet has been designed to facilitate its use. We plan to use it in remote places with telementoring concepts.

No competing financial interests exist.

Runtime of video: 5 mins

Keywords: simulation model for MIS training, pediatric MIS training model, low-cost simulation model for pediatric MIS

\section{Cite this video}

Maria M. Bailez, Georgina Falcioni, Hsien Yang, Maximiliano Maricic, Patricia Martinez, Carolina Ruiz, Roxana Colombo, Pablo Schiavo, Developing a Gruyere-Like Trainer to Use in the Transition Between Basic and Neonatal Simulation-Based Minimally Invasive Surgery Education, Videoscopy. 2019, DOI: 10.1089/vor.2019.0583.

Original Publication Date: 2019 